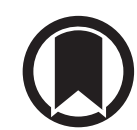

CrossMark

\title{
Tolerability of rifapentine-based regimens in latent tuberculosis infection treatment in the elderly
}

\author{
To the Editor:
}

Management of latent tuberculosis infection (LTBI) is a core intervention in the pursuit of tuberculosis (TB) elimination and is one of the eight activities recommended by the World Health Organization [1]. We read with great interest the paper by GAO et al. [2] investigating two short-course regimens with rifapentine plus isoniazid for the treatment of LTBI in Chinese patients aged 50-70 years. They reported that due to the ever-increasing occurrence of adverse events, the 3-month once-weekly regimen and the 2-month twice-weekly regimen were truncated to 8 and 6 weeks, respectively. GAO et al. [2] concluded that the short regimens tested must be used with caution among the elderly because of a high frequency of adverse events. The findings reported by GAO et al. [2] were not consistent with our experience in Taiwan.

The use of 3-month once-weekly rifapentine plus isoniazid (3HP) for the treatment of LTBI in Taiwan began with two pilot studies that enrolled contacts of TB patients $[3,4]$. Subsequently, the LTBI Treatment for All Contacts Program was initiated in 2016, providing 3HP as well as 9-month-isoniazid (9H) for the treatment of LTBI among contacts aged 12 years or older with directly observed preventive therapy. $3 \mathrm{HP}$ was endorsed by the Taiwan Centers for Disease Control [5], although at that time rifapentine was not yet approved by the Taiwan Food and Drug Administration [6].

We closely monitored adverse events and reasons for the discontinuation of LTBI treatment were entered into the electronic TB case management system by public health nurses. Serious adverse events were defined as any hospitalisation, emergency department visit for more than $24 \mathrm{~h}$ or death during treatment. We reviewed case management records and medical charts to estimate serious adverse events associated with 3HP using the Modified Naranjo Adverse Drug Reaction Probability Scale [7].

Between April 2016 and June 2018, a total of 14676 individuals received treatment of LTBI, of whom 7974 (54\%) were aged 50 years or older. Of the 7974 individuals, 5087 (63.8\%) preferred 3HP and 2887 preferred $9 \mathrm{H}$. The proportion of individuals with early termination of treatment due to adverse events in those who were treated with $3 \mathrm{HP}$ was lower than that with those treated with $9 \mathrm{H}(8.0 \%(409 / 5087)$ versus 9.4\% (272/2887); $\mathrm{p}=0.03)$. The most frequently encountered adverse events leading to permanent discontinuation differed between the two regimens. Among patients who terminated $3 \mathrm{HP}$ early, 30\% discontinued treatment due to fever; among patients who terminated $9 \mathrm{H}$ early, $53 \%$ discontinued due to drug-induced hepatitis. 85 (0.84\%) patients treated with $3 \mathrm{HP}$ had serious adverse events and permanently discontinued 3HP, mainly due to flu-like illness (53\%), hypersensitivity (24\%) and hepatotoxicity (18\%). No death or long-term sequelae associated with $3 \mathrm{HP}$ was observed.

We analysed completion of LTBI treatment among 4172 individuals aged 50 years and older who had LTBI treatment initiated between April 2016 and July 2017 (table 1). Those who received 3HP had a higher treatment completion rate compared to those receiving $9 \mathrm{H}(83.9 \%$ versus $78.8 \%$; $<<0.001)$. GAO et al. [2] reported that $1 \%$ of those who received $3 \mathrm{HP}$ had drug-induced hepatotoxicity leading to permanent discontinuation of treatment after 8 weeks. This figure was found to be $0.8 \%$ in our study cohort and no fulminant hepatic failure was noted.

@ERSpublications

Our experience has shown that with proper support and medical care under programmatic conditions, it is feasible to achieve a high completion rate of $3 \mathrm{HP}$ even among the elderly http://ow.ly/ o3ED30nQfRx

Cite this article as: Chan P-C, Lee P-H, Lu M-J, et al. Tolerability of rifapentine-based regimens in latent tuberculosis infection treatment in the elderly. Eur Respir J 2019; 53: 1802396 [https://doi.org/10.1183/ 13993003.02396-2018]. 
TABLE 1 Treatment outcome of latent tuberculosis infection for those aged 50 years and older

\begin{tabular}{lccc} 
& 3HP & 9H & p-value \\
\hline Total subjects & $2348(100 \%)$ & $1824(100 \%)$ & \\
Males & $1129(48.1 \%)$ & $839(46.0 \%)$ & 0.181 \\
Age $<75$ years & $2054(87.5 \%)$ & $1314(72.0 \%)$ & $<0.001$ \\
$\begin{array}{l}\text { Treatment completed } \\
\text { Any adverse event leading to permanent } \\
\quad \text { discontinuation of treatment }\end{array}$ & $1971(83.9 \%)$ & $1438(78.8 \%)$ & $<0.001$ \\
$\begin{array}{l}\text { Hepatotoxicity leading to permanent } \\
\text { discontinuation of treatment }\end{array}$ & $18(12.0 \%)$ & $172(9.4 \%)$ & 0.009 \\
\end{tabular}

Total $n=4172$. $p$-values are calculated using the Chi-square test. 3HP: 3-month once-weekly rifapentine plus isoniazid; 9H: 9-month isoniazid.

Drug quality may be associated with adverse events. Currently, isoniazid used in Taiwan is generic and is domestically produced by pharmaceutic companies with good manufacturing practice following a pharmaceutical inspection co-operation scheme. Rifapentine (Priftin) was purchased from Sanofi produced in Italy. Since the study was discontinued early, we wonder to what extent a high frequency of adverse events observed by GAO et al. [2] were due to the drugs that they used among rural residents in Zhongmu County, China. Furthermore, tolerability of treatment of LTBI could be relatively low because the subjects in Zhongmu did not have clinical symptoms as severe as hepatic failure. GAO et al. [2] did not use $9 \mathrm{H}$ and were not able to assess tolerability of $9 \mathrm{H}$ in their study population. Thus, it is not clear to what extent tolerability of LTBI treatment itself has contributed to the challenge of completing $3 \mathrm{HP}$ treatment.

It has been reported that age is associated with adverse events and the elderly are more likely to experience adverse events during the treatment of LTBI, regardless of the regimens used $[4,8]$. However, our experience has shown that with proper support and medical care under programmatic conditions, it is feasible to achieve a high completion rate of 3HP, even among the elderly [4, 9]. Treatment of LTBI among the elderly to mitigate the risk of developing active TB is crucial in settings where TB burden is disproportionally high among the elderly [10]. Our experience shows that the use of $3 \mathrm{HP}$ for the treatment of LTBI is a useful tool for achieving the goals of the "The End TB Strategy" and, hopefully, in eliminating TB.

Pei-Chun Chan ${ }^{1,2,3}$, Pin-Hui Lee, Min-Ju Lu ${ }^{1}$, You-Chieh Huang ${ }^{1}$, Teng-Yi Feng ${ }^{1}$, Wen-Wen Chen ${ }^{4}$, Chia-Chi Lee ${ }^{1}$, Yen-Fang Huang ${ }^{1}$ and Chen-Yuan Chiang ${ }^{5,6,7}$

${ }^{1}$ Division of Chronic Infectious Disease, Centers for Disease Control, Taipei, Taiwan. ${ }^{2}$ Institute of Epidemiology and Preventive Medicine, College of Public Health, National Taiwan University, Taipei, Taiwan. ${ }^{3}$ Dept of Pediatrics, National Taiwan University Hospital, National Taiwan University, College of Medicine, Taipei, Taiwan. ${ }^{4}$ Taiwan Drug Relief Foundation, Taipei, Taiwan. ${ }^{5}$ Division of Pulmonary Medicine, Dept of Internal Medicine, Wanfang Hospital, Taipei Medical University, Taipei, Taiwan. ${ }^{6}$ Division of Pulmonary Medicine, Dept of Internal Medicine, School of Medicine, College of Medicine, Taipei Medical University, Taipei, Taiwan. ${ }^{7}$ International Union against Tuberculosis and Lung Disease, Paris, France.

Correspondence: Pei-Chun Chan, Division of Chronic Infectious Disease, Centers for Disease Control, Taiwan, 6 Linshen South Road, Taipei 100, Taiwan. E-mail: pcanita.tw@cdc.gov.tw

Received: Dec 172018 | Accepted after revision: Feb 142019

Conflict of interest: None declared.

\section{Reference}

1 Lönnroth K, Migliori GB, Abubakar I, et al. Towards tuberculosis elimination: an action framework for low-incidence countries. Eur Respir J 2015; 45: 928-952.

2 Gao L, Zhang H, Xin H, et al. Short-course regimens of rifapentine plus isoniazid to treat latent tuberculosis infection in older Chinese patients: a randomised controlled study. Eur Respir J 2018; 52: 1801470.

3 Huang YW, Yang SF, Yeh YP, et al. Impacts of 12-dose regimen for latent tuberculosis infection: Treatment completion rate and cost-effectiveness in Taiwan. Medicine (United States) 2016; 95: e4126.

4 Sun HY, Huang YW, Huang WC, et al. Twelve-dose weekly rifapentine plus isoniazid for latent tuberculosis infection: a multicentre randomised controlled trial in Taiwan. Tuberculosis (Edinb) 2018; 111: 121-126.

5 Centers for Disease Control and Prevention. Recommendations for use of an isoniazid-rifapentine regimen with direct observation to treat latent Mycobacterium tuberculosis infection. MMWR Morb Mortal Wkly Rep 2011; 60: 1650-1653.

6 Food and Drug Administration, Ministry of Health and Welfare, Taiwan. The content of license for Priftin 150mg film-coated tablets. www.fda.gov.tw/MLMS/H0001D.aspx?Type=Lic\&LicId=52027203 Date last updated: July 28, 2017. Date last accessed: Feb 11, 2019. 
7 Sterling TR, Moro RN, Borisov AS, et al. Flu-like and other systemic drug reactions among persons receiving weekly rifapentine plus isoniazid or daily isoniazid for treatment of latent tuberculosis infection in the PREVENT tuberculosis study. Clin Infect Dis 2015; 61: 527-535.

8 Kunst H, Khan KS. Age-related risk of hepatotoxicity in the treatment of latent tuberculosis infection: a systematic review. Int J Tuberc Lung Dis 2010; 14: 1374-1381.

9 Sandul AL, Nwana N, Holcombe JM, et al. High rate of treatment completion in program settings with 12-dose weekly isoniazid and rifapentine for latent Mycobacterium tuberculosis infection. Clin Infect Dis 2017; 65: 1085-1093.

10 Houben RM, Dodd PJ. The global burden of latent tuberculosis infection: a re-estimation using mathematical modelling. PLoS Med 2016; 5: 1-13.

Copyright @ERS 2019 